\title{
On Symmetrical Deformation of Toroidal Shell with Circular Cross-Section
}

\author{
Bo-Hua SUN ${ }^{1}$ \\ ${ }^{1}$ Institute of Mechanics and Technology \& School of Civil Engineering, \\ Xi'an University of Architecture and Technology, Xi'an 710055, China \\ http://imt.xauat.edu.cn \\ email: sunbohua@xauat.edu.cn
}

\begin{abstract}
By introducing a variable transformation $\xi=\frac{1}{2}(\sin \theta+1)$, the symmetrical deformation equation of elastic toroidal shells is successfully transferred into a well-known equation, namely Heun's equation of ordinary differential equation, whose exact solution is obtained in terms of Heun's functions. The computation of the problem can be carried out by symbolic software that is able to with the Heun's function, such as Maple. The Gauss curvature of the elastic toroidal shells shows that the internal portion of the toroidal shells has better bending capacity than the outer portion, which might be useful for the design of metamaterials with toroidal shells cells. Through numerical comparison study, the mechanics of elastic toroidal shells is sensitive to the radius ratio. By slightly adjustment of the ratio might get a desired high performance shell structure.
\end{abstract}

Keywords: toroidal shells, deformation, Gauss curvature, Heun's function, hypergeometric function, Mathieu function, Maple

\section{INTRODUCTION}

Among of most regular shell, such as circular cylindrical shells, conical shells, spherical shells and toroidal shells, the deformation of toroidal shells is one of upmost difficulty problem due to its complicated topology. Up to date, its exact solution at general radius ration has not been obtained yet.

Toroidal shells, in full or partial geometric form as shown in Figures 1, is widely used in structural engineering and have been extensively investigated [1-20].
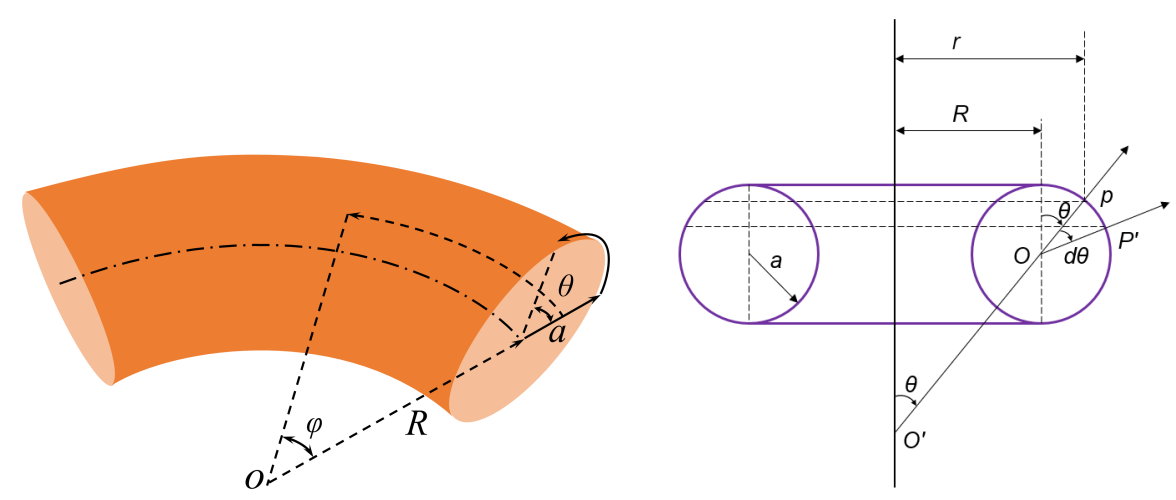

FIG. 1: Toroidal shells and cross-sectional view. Toroidal shells and geometry. The principal radius of curvature $R_{1}=a$ and $R_{2}=a+\frac{R}{\sin \theta}$; the principal curvature $K_{1}=\frac{1}{a}, K_{2}=\frac{\sin \theta}{R+a \sin \theta}$; the Gauss curvature $K=K_{1} K_{2}=\frac{\sin \theta}{a(R+a \sin \theta)}$.

The difficulty source of finding solution comes from the geometric feature of the toroidal shells. The toroidal shells's Gauss curvature $K$ changes its sign as principal radius of curvature $R_{\theta}$ when the angle $\theta$ goes from 0 to $2 \pi$, it means that Gauss curvature has a turning point, namely $K=0$ at $\theta=\pi$ as shown in Fig. 1. Recall that the partial differential equations governing the elasticity of elliptic shells $K>0$ are themselves elliptic while those for hyperbolic shells $K<0$ are hyperbolic. This means that the equations for a toroidal shell are of a mixed type, namely elliptic in the outer half of the toroidal shells, and hyperbolic in the inner one. The exist of the turning point in a complete toroidal shells is one source of the difficulty to find a solution. Owing to the difficulty of solving partial differential 


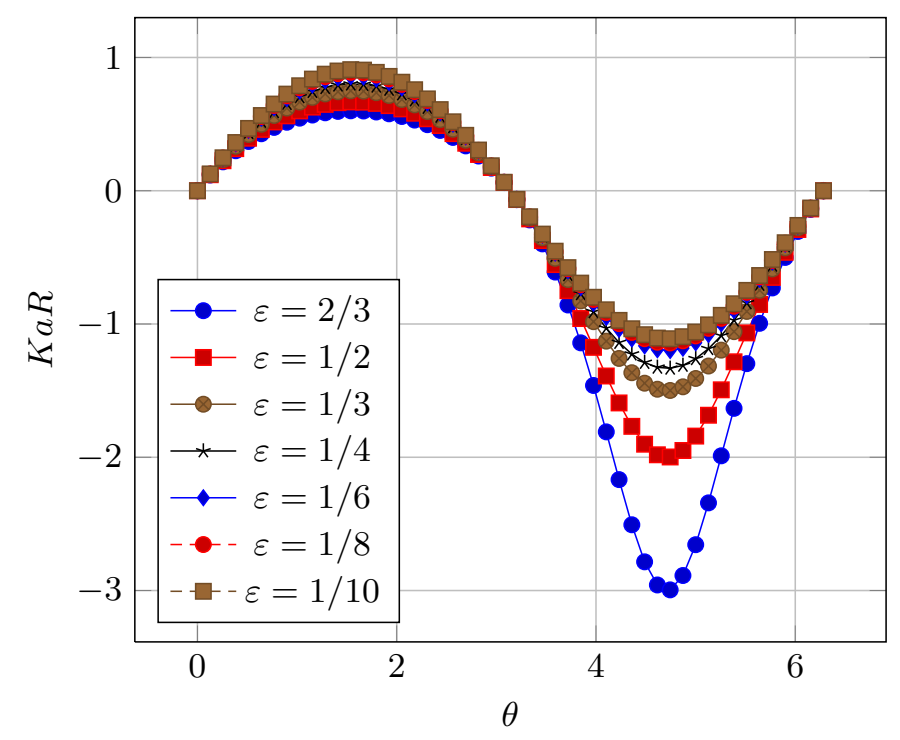

FIG. 2: The curves of $R a K=\frac{\sin \theta}{1+\varepsilon \sin \theta}$, where $\varepsilon=\frac{a}{R}$. The geometry of toroidal shells surface is elliptic in $\theta \in[0, \pi]$, parabolic at $\theta=0$ and hyperbolic $\theta \in[0,-\pi]$.

equations with both hyperbolic and elliptic regions, few general results are known for shells with mixed type [15-19]. To find the solution of elastic toroidal shells still remains a great challenge.

The Gauss curvature change sign also provides some special feature of mechanics. Since the bending capacity of the shell is proportional to the Gauss curvature, the Fig. 2 reveals that the inner part of toroidal shells $(\theta \in[\pi, 2 \pi])$ is much stronger than outer part of the shell $(\theta \in[0, \pi])$. Therefore, for given amount of materials, to construct a high bending performance toroidal shells, the internal toroidal shells topology is the best choice. This secret mechanical performance might be useful to the design of metamaterials with toroidal shells cell.

To attack the problem, the high order and complicated governing equations of toroidal shells under symmetric loads is reduced to a single equation of lower order complex equation. The complex form governing equations of toroidal shells of revolution were formulated firstly by Reissner (1912)[1], Wissller (1916) [3] and later finalized by Novozhilov (1959) [7] a follows:

$$
(1+\varepsilon \sin \theta) \frac{d^{2} V}{d \theta^{2}}-\varepsilon \cos \theta \frac{d V}{d \theta}+2 i d^{2} \sin \theta V=P(\theta)
$$

Eq. 1 is called Reissner-Novozhilov equation of toroidal shells, in which, $P(\theta)=-2 d^{2}\left(2 d^{2} A+\frac{1}{2} i \varepsilon q a\right) \cos \theta, 2 d^{2}=$ $\frac{a^{2}}{R h} \sqrt{12\left(1-\mu^{2}\right)}, \mu$ is the Poisson's ratio, $h$ is thickness, and $q$ is distributed loads, $A$ is an integration constant.

Beside the turning point issue, it is clear that the challenge is also comes from the variable coefficients of the differential equation in Eq.1.

Nevertheless, some kind solutions have been proposed successfully. The first exact series solution of toroidal equations was obtained by Wissler (1916) [3], however, Wissler's series solution has little practical value due to its slow convergency and not established a linkage with any special known functions as well.

To provide practical solution, thus a various of asymptotic solutions have been proposed [14-16], however, the turning point of the Gauss curvature makes all proposed asymptotic solutions invalid near the point. Up to date, no exact solution in terms of special functions has been obtained for symmetrical deformation of toroidal shells, except in the case of slender toroidal shells, whose displacement type closed-form solution is obtained by Sun (2011) [17, 19].

To find an exact solution that can be expressed in a special function is still a open question even after more than centenary development of the theory of toroidal shells. In this paper, we will shoulder this historical responsibility and propose an exact solution in terms of special functions. Once we obtain the solution in terms of well-known special functions, the convergency issue will be solved. 
The paper is organized into various sections, namely: Section 2 finds exact solution; Section 3 presents two numerical cases; Section IV, finally, Section 4 concludes with future perspectives.

\section{EXACT SOLUTION OF SYMMETRICAL DEOFRMATION OF TOROIDAL SHELLS OF REVOLUTION}

To solve the Eq.1, Wissler [3] introduced a variable transformation, $x=\sin \theta$, thus leads $d x=\cos \theta d \theta, \frac{d V}{d \theta}=$ $\frac{d V}{d x} \frac{d x}{d \theta}=\cos \theta \frac{d V}{d x}$ and $\frac{d^{2} V}{d \theta^{2}}=\cos ^{2} \theta \frac{d 2 V}{d x^{2}}-\sin \theta \frac{d V}{d x}=\left(1-x^{2}\right) \frac{d^{2} V}{d x 2}-x \frac{d V}{d x}$, hence the Eq.1 is transferred into following form

$$
\left(1-x^{2}\right)(1+\varepsilon x) \frac{d^{2} V}{d x^{2}}-(x+\varepsilon) \frac{d V}{d x}+2 i d^{2} x V=P(x),
$$

where $P(x)=-2 d^{2}\left(2 d^{2} A+\frac{1}{2} i \varepsilon q a\right) \sqrt{1-x^{2}}$. The Eq.2 is a Fuchian type differential equation whose series solution was given by Wissler [3].

In order to establish Eq.2 with well-known equations, let's carry on and introducing another variable transformation,

$$
\xi=\frac{1}{2} x+\frac{1}{2}=\frac{1}{2}(\sin \theta+1)
$$

thus $\frac{d V}{d x}=\frac{1}{2} \frac{d V}{d \xi}, \frac{d^{2} V}{d x^{2}}=\frac{1}{4} \frac{d^{2} V}{d \xi^{2}}$, and $1-x^{2}=-4 \xi(\xi-1)$, hence Eq.2 can be transferred into

$$
\xi(\xi-1)\left[\xi-\left(\frac{1}{2}-\frac{1}{2 \varepsilon}\right)\right] \frac{d^{2} V}{d \xi^{2}}+\frac{1}{2 \varepsilon}\left(\xi-\frac{1}{2}+\frac{\varepsilon}{2}\right) \frac{d V}{d \xi}+\left(-\frac{4 i d^{2}}{\varepsilon} \xi+\frac{i d^{2}}{\varepsilon}\right) V=P(\xi),
$$

where $P(\xi)=\frac{2 d^{2}}{\varepsilon}\left(2 d^{2} A+\frac{1}{2} i \varepsilon q a\right) \sqrt{\xi(1-\xi)}$; and or in another popular format as follows

$$
\frac{d^{2} V}{d \xi^{2}}+\left(\frac{\frac{1}{2}}{\xi}+\frac{\frac{1}{2}}{\xi-1}+\frac{-1}{\xi-\frac{\varepsilon-1}{2 \varepsilon}}\right) \frac{d V}{d \xi}+\frac{-\frac{2 i d^{2}}{\varepsilon} \xi+\frac{i d^{2}}{\varepsilon}}{\xi(\xi-1)\left(\xi-\frac{\varepsilon-1}{2 \epsilon}\right)} V=\frac{2 d^{2}}{\varepsilon} \frac{2 d^{2} A+\frac{1}{2} i \varepsilon a q}{\xi(\xi-1)\left(\xi-\frac{\varepsilon-1}{2 \varepsilon}\right)}
$$

The Eq.4 and/or 5 is a Fuchian type differential equation that has been studied by Heun (1889) [21]. Eq.4 is called general Heun's equation, whose solutions can be represented by the Heun's functions. It is clear that the numerical advantage of solution being expressed by Heun's functions is that software package able to work with the Heun functions, such as MAPLE [25], can be used for calculations, which by the way will sort out the convergency issue of the solution.

The Heun functions (named after Karl Heun: 1859-1929) are unique local Frobenius solutions of a second-order linear ordinary differential equation of the Fuchsian type which in the general case have 4 regular singular points. Heun's equation is an extension of the ${ }_{2} F_{1}$ hypergeometric equation in that it is a second-order Fuchsian equation with four regular singular points. The ${ }_{2} F_{1}$ equation has three regular singularities. The HeunG function, thus, contains as particular cases all the functions of the hypergeometric ${ }_{2} F_{1}$ class [21-23].

The Heun functions generalize the hypergeometric function, the Láme function, Mathieu function [24] and the spheroidal wave functions. Because of the wide range of their applications, they can be considered as the 21st century successors of the hypergeometric functions. [25].

The exact solution of Eq.4 is summation of homogenous solution $V^{h}(x)$ and particular solution $V^{p}(x)$, namely, $V=V^{h}+V^{p}$, both of them can be expressed by Heun functions. The homogenous solution can be given as follows

$$
V^{h}(x)=C_{1} y_{1}(x)+C_{2}(x+1)^{\frac{1}{2}} y_{2}(x),
$$

where

$$
\begin{aligned}
& y_{1}(x)=H \operatorname{eun} G\left(\frac{\varepsilon-1}{2 \varepsilon},-\frac{i d^{2}}{\varepsilon},-\frac{1}{2} \frac{\sqrt{\varepsilon} \sqrt{8 i d^{2}+\varepsilon}+\varepsilon}{\varepsilon}, \frac{1}{2} \frac{8 i d^{2}+\sqrt{\varepsilon} \sqrt{8 i d^{2}+\varepsilon}-\varepsilon}{\sqrt{\varepsilon} \sqrt{8 i d^{2}+\varepsilon}+2 \varepsilon}, \frac{1}{2}, \frac{1}{2}, \frac{1}{2}(x+1)\right), \\
& y_{2}(x)=\operatorname{Heun} G\left(\frac{\varepsilon-1}{2 \varepsilon},-\frac{1}{8} \frac{8 i d^{2}+3 \varepsilon+1}{\varepsilon},-\frac{1}{2} \frac{\sqrt{8 i d^{2}+\varepsilon}}{\sqrt{\varepsilon}}, \frac{1}{2} \frac{8 i d^{2}+2 \sqrt{\varepsilon} \sqrt{8 i d^{2}+\varepsilon}+\varepsilon}{\sqrt{\varepsilon} \sqrt{8 i d^{2}+\varepsilon}+2 \varepsilon}, \frac{3}{2}, \frac{1}{2}, \frac{1}{2}(x+1)\right) .
\end{aligned}
$$


where the general Heun's function HeunG can be computed for given loading condition.

Substitutes the Wissler's transformation $x=\sin \theta$ into the above solutions, we have

$$
V^{h}(\theta)=C_{1} y_{1}(\theta)+C_{2}(\sin \theta+1)^{\frac{1}{2}} y_{2}(\theta)
$$

where

$$
\begin{aligned}
& y_{1}(\theta)=\operatorname{Heun} G\left(\frac{\varepsilon-1}{2 \varepsilon},-\frac{i d^{2}}{\varepsilon},-\frac{1}{2} \frac{\sqrt{\varepsilon} \sqrt{8 i d^{2}+\varepsilon}+\varepsilon}{\varepsilon}, \frac{1}{2} \frac{8 i d^{2}+\sqrt{\varepsilon} \sqrt{8 i d^{2}+\varepsilon}-\varepsilon}{\sqrt{\varepsilon} \sqrt{8 i d^{2}+\varepsilon}+2 \varepsilon}, \frac{1}{2}, \frac{1}{2}, \frac{1}{2}(\sin \theta+1)\right), \\
& y_{2}(\theta)=\operatorname{Heun} G\left(\frac{\varepsilon-1}{2 \varepsilon},-\frac{1}{8} \frac{8 i d^{2}+3 \varepsilon+1}{\varepsilon},-\frac{1}{2} \frac{\sqrt{8 i d^{2}+\varepsilon}}{\sqrt{\varepsilon}}, \frac{1}{2} \frac{8 i d^{2}+2 \sqrt{\varepsilon} \sqrt{8 i d^{2}+\varepsilon}+\varepsilon}{\sqrt{\varepsilon} \sqrt{8 i d^{2}+\varepsilon}+2 \varepsilon}, \frac{3}{2}, \frac{1}{2}, \frac{1}{2}(\sin \theta+1)\right) .
\end{aligned}
$$

After determination of the auxiliary function $V(\theta)$, all other quantities can be expressed as by the function $V(\theta)$, such as middle surface resultant force $T_{1}, T_{2}$

$$
\begin{aligned}
& T_{1}=-\frac{\varepsilon \cos \theta}{2 d^{2}(1+\varepsilon \sin \theta)} \operatorname{Im}(V)+\frac{q a}{2} \frac{2+\varepsilon \sin \theta}{1+\varepsilon \sin \theta}-\varepsilon A \frac{\varepsilon+\sin \theta}{(1+\varepsilon \sin \theta)^{2}}, \\
& T_{2}=-\frac{1}{2 d^{2}} \operatorname{Im}\left[\frac{d}{d \theta}\left(\frac{V}{1+\varepsilon \sin \theta}\right)\right]+\frac{q a}{2}+\varepsilon A \frac{\varepsilon+\sin \theta}{(1+\varepsilon \sin \theta)^{2}},
\end{aligned}
$$

resultant moments $M_{1}$

$$
M_{1}=-\frac{h}{2 d^{2} \sqrt{12(1-\mu)}} \frac{\mu \varepsilon \cos \theta}{(1+\varepsilon \sin \theta)^{2}} \operatorname{Re}(V)-\frac{h}{2 d^{2} \sqrt{12(1-\mu)}} \operatorname{Re}\left[\frac{d}{d \theta}\left(\frac{V}{1+\varepsilon \sin \theta}\right)\right] .
$$

and resultant shear force $N_{1}$

$$
N_{1}=-\frac{h}{a \sqrt{12\left(1-\mu^{2}\right)}} \frac{\sin \theta \operatorname{Im}(V)+2 d^{2} A \cos \theta}{(1+\varepsilon \sin \theta)^{2}}
$$

\section{NUMERICAL CASE STUDIES OF SYMMETRICAL DEFORMATION OF TOROIDAL SHELLS}

A complete toroidal shells with a penetrate cut along the parallel $\theta=\frac{\pi}{2}\left(\right.$ or $\left.\theta=-\frac{\pi}{2}\right)$ and loaded with distributed bending moment $M_{0}$, as shown in Fig.3

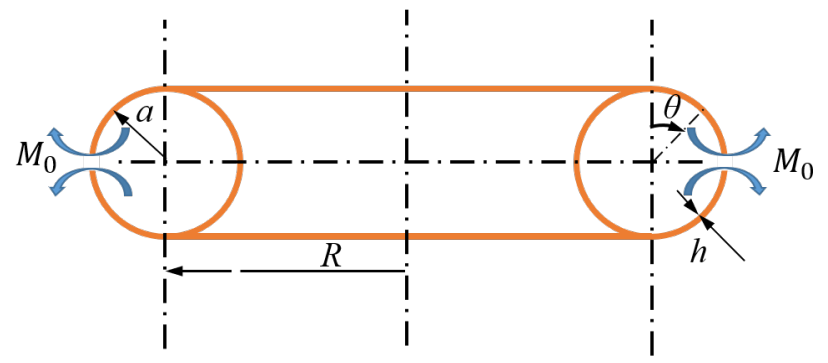

FIG. 3: Toroidal shells with a cut along its parallel at $\theta=\frac{\pi}{2}$ or $\theta=-\frac{\pi}{2}$ under a pure moment $M_{0}$

The toroidal shells's geometric and material properties data are listed in table below:

TABLE I: Data of slender toroidal shells

\begin{tabular}{c|c|c|c|c|c}
\hline$R$ & $a$ & $h$ & $E$ & $\mu$ & $M_{0}$ \\
\hline \hline $\mathrm{m}$ & $\mathrm{m}$ & $\mathrm{m}$ & $N / m^{2}$ & & $N$ \\
\hline 1 & 0.1 & 0.0004 & $2.07 \times 10^{11}$ & 0.3 & $M_{0}$ \\
\hline
\end{tabular}


The bending stiffness $D=\frac{E h^{3}}{12\left(1-\mu^{2}\right.}=1.213186813 \times 10^{6}$ and middle surface stiffness $K=\frac{E h}{1-\mu^{2}}=9.098901099 \times 10^{9}$. The boundary is:

$$
\varphi=\frac{\pi}{2}: M_{1}=M_{0}, N_{1}=0, T_{1}=0, T_{2}=0
$$

Under the above loading and boundary condition, the constant of integration $A=0$ and distributed load $q=0$.

\section{Case 1: Slender toroidal shells with the radius ratio $\left(\varepsilon=\frac{a}{R}=0\right)$}

To demonstrate the analytical results. let's apply to a slender toroidal shells, namely $\varepsilon=0$, with bending moment loading $M_{0}$. The governing equation can be reduced further to

$$
\left(1-x^{2}\right) \frac{d^{2} V}{d x^{2}}-x \frac{d V}{d x}+i \frac{a \sqrt{12\left(1-\mu^{2}\right)}}{R h} x V=0,
$$

whose solution is given by

$$
V(\theta)=C_{1} \text { MathieuC }\left(0, \frac{413067791}{5}, \frac{1}{2} \theta-\frac{\pi}{4}\right)+C_{2} \text { MathieuS }\left(0, \frac{413067791}{5}, \frac{1}{2} \theta-\frac{\pi}{4}\right),
$$

where the transformation $x=\sin \theta$ has been used. With the obtained $V(\theta)$, we can compute other quantifies, such as

$$
\begin{aligned}
& M_{1}=-\frac{R h^{2}}{12 a^{2}\left(1-\mu^{2}\right)} \operatorname{Re}\left(\frac{d V}{d \theta}\right), \quad N_{1}=-\frac{h \sin \theta}{a \sqrt{12\left(1-m u^{2}\right)}} \operatorname{Im}(V), \\
& T_{1}=0, \quad T_{2}=\frac{R h \sqrt{12\left(1-\mu^{2}\right)}}{a^{2}} \operatorname{Im}(V),
\end{aligned}
$$

where, Re and $I m$ are real and imaginary part of a complex. Applying the boundary condition in Eq.16, we can find $C_{1}=0$ and $C_{2}=-34.125 M_{0}$

Using Maple [25] to compute the Mathieu's functions, we can get the resultant bending moment $M_{1}$ and resultant shear force $N_{1}$ as follows

$$
\begin{aligned}
& M_{1}=M_{0} \operatorname{Re}\left(\text { MathieuSPrime }\left(0,8.2613558 i, 0.785398+\frac{1}{2} \theta\right),\right) \\
& N_{1}=8.26 M_{0} \sin \theta \operatorname{Im}\left(\text { MathieuS }\left(0,8.2613558 i, 0.785398+\frac{1}{2} \theta\right)\right), \\
& T_{2}=902.14 M_{0} \operatorname{Im}\left(\text { MathieuSPrime }\left(0,8.2613558 i, 0.785398+\frac{1}{2} \theta\right)\right) .
\end{aligned}
$$

The resultant bending moment $M_{1}$ and resultant shear force $N_{1}$ are plotted in Fig. 4 as below.

The solution of the slender toroidal shells increase dramatically in the range of $\left[-\frac{\pi}{2}, \frac{\pi}{8}\right]$, the slender solution is not valid in the domain, which implies that slender model might be not a proper approximation of toroidal shells.

\section{Case 2: Toroidal shells with the radius ratio $\left(\varepsilon=\frac{a}{R}=\frac{1}{20}\right)$}

In order to see the influence of the radius ratio $\varepsilon=\frac{a}{R}$, let's investigate a case of $\varepsilon=\frac{1}{20}$. In this case, the governing equation is given as

$$
\left(1-x^{2}\right)(1+\varepsilon x) \frac{d^{2} V}{d x^{2}}-(x+\varepsilon) \frac{d V}{d x}+i \frac{a^{2}}{R h} \sqrt{12\left(1-\mu^{2}\right)} x V=0
$$

whose solution is obtained as follows

$$
V(\theta)=C_{1} V_{1}+2 \sqrt{2} C_{2} \cos \theta V_{2} .
$$



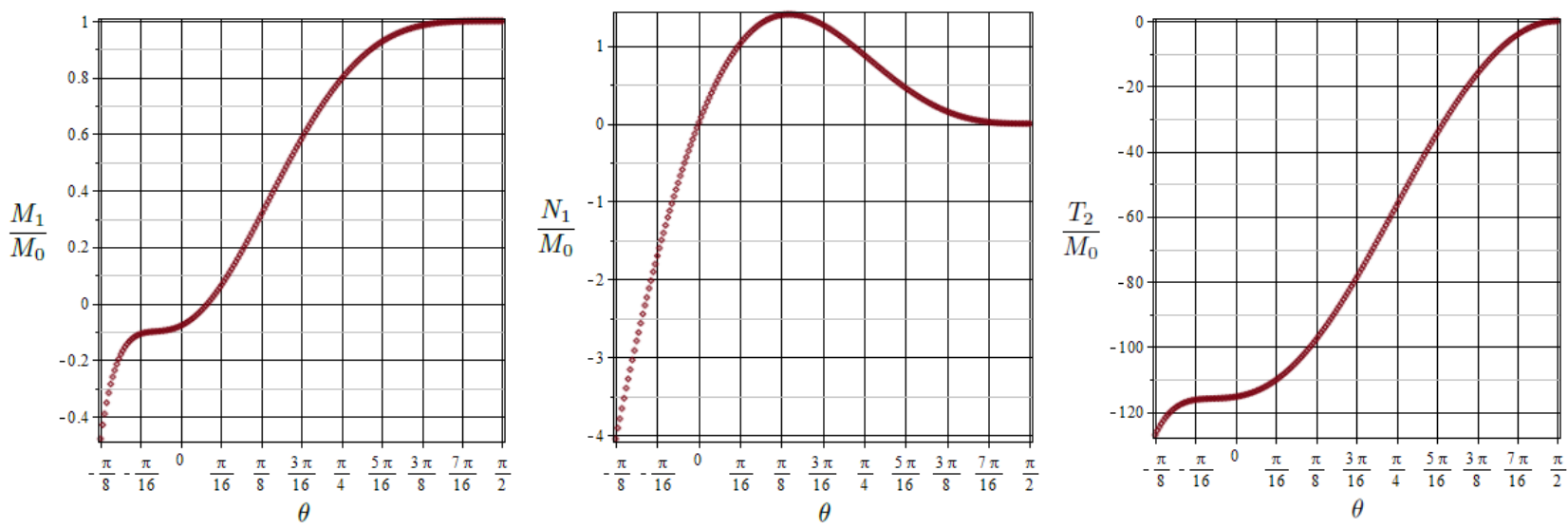

FIG. 4: Case $\varepsilon=0$ : The resultant bending moment $M_{1}$, the resultant shear force $N_{1}$ and the resultant middle-surface force $T_{2}$ vs $\theta$.

where $V_{1}=H e u n G\left(-9.5,-41.307 i, 5.937+6.4173 i,-6.937-6.417 i, \frac{1}{2}, \frac{1}{2}, \frac{1}{2} \sin \theta+\frac{1}{2}\right)$ and $V_{2}=H e u n G(-9.5,-2.875-$ $\left.41.307 i, 6.437+6.417 i,-6.437-6.417 i, \frac{3}{2}, \frac{1}{2}, \frac{1}{2} \sin \theta+\frac{1}{2}\right)$. The constants of integration $C_{1}$ and $C_{2}$ can be determined by the boundary, $C_{1}=0$ and $C_{2}=2.9109 M_{0}$. All other quantities can be obtained accordingly. Due to length of their expressions, we only give $T_{1}$ as follows

$$
T_{1}=-5.8218 \sqrt{2} M_{0} \frac{\cos \theta}{1+\frac{1}{2} \sin \theta} \operatorname{Im}\left(V_{2}\right) .
$$

For the case of $\varepsilon=\frac{1}{20}$, the resultant bending moment $M_{1}$, the resultant shear force $N_{1}$ and the resultant middle-surface force $T_{1}$ vs $\theta$ are plotted in Fig.5 below.
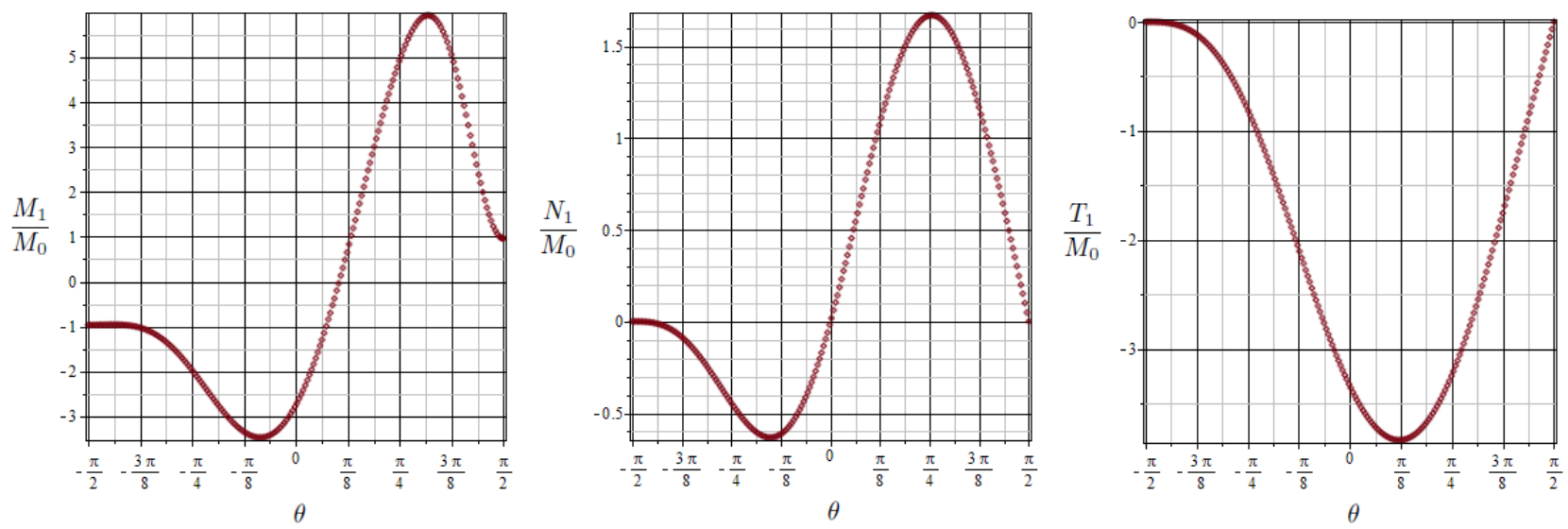

FIG. 5: Case $\varepsilon=\frac{1}{20}$ : The resultant bending moment $M_{1}$, the resultant shear force $N_{1}$ and the resultant middle-surface force $T_{1}$ vs $\theta$.

With slight change of the radius ration from 0 to $\frac{1}{20}$, the solution curves in the above figures are much improved, which reveals that the radius ration $\varepsilon$ is a vital parameter for .

\section{Comparison study of two case}

To evaluate the validity of of slender model od toroida shell, some kind of comparison studies should be carried. For instance, the bending moments of the shells $M_{1}$ are plotted in Fig.6. The comparisons strongly indicate the slender 

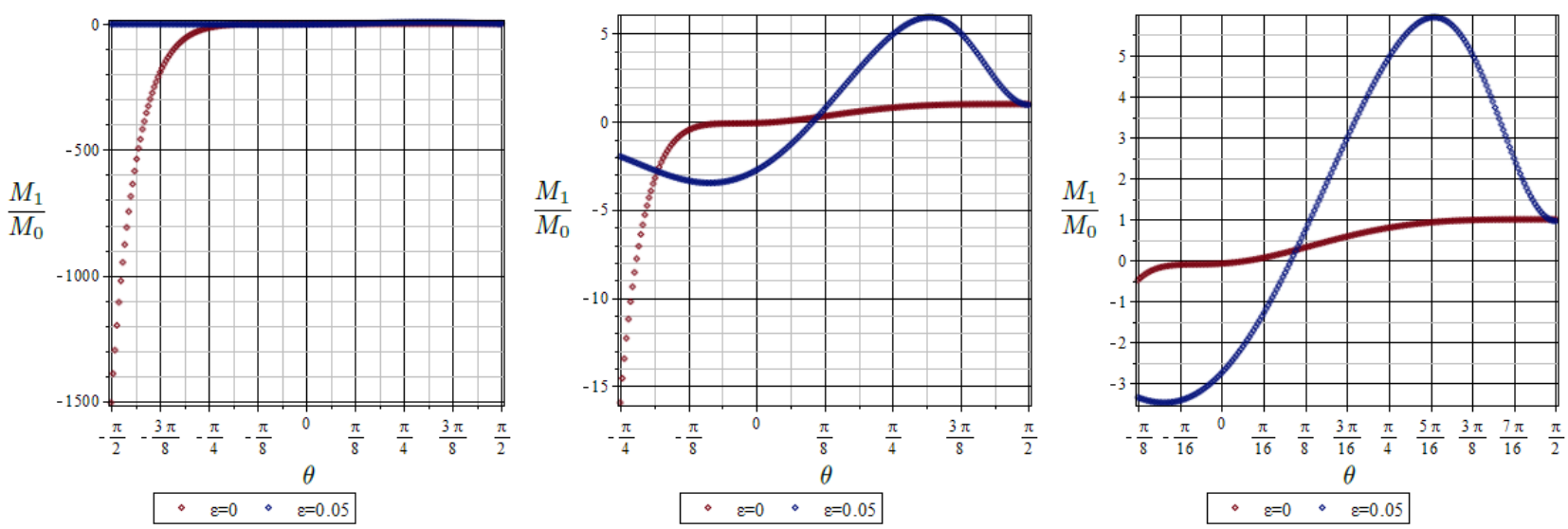

FIG. 6: Comparison study for the resultant bending moment $M_{1}$ as $\varepsilon$ varies

model is questionable and must be use with care. In other words, the deformation feature of the toirdal shells are quite sensitive to the radius ratio $\varepsilon$.

This geometrical-mechanics sensitively can be utilised in the design of the shells, ie., the desired mechanics of the shells can be archived by slightly change of the radius ratio $\varepsilon$.

\section{SUMMARY AND CONCLUSIONS}

We have successfully obtained exact solution of complex form equation for symmetrical deformation of toroidal shells. The research has confirmed that the deformation of all regular shell structure, such cylindrical shell, conical shell, spherical shell and toiroidal shell can be solved by hypergeometric functions. This supports the doctrine of Zurich school of shell theory $[1,2,7]$, which predicted that bending deformation of all regular shells can be expressed by the hypergeometric functions. Through numerical comparison study, the mechanics of toroidal shells is quite sensitive to the radius ratio $\varepsilon=\frac{a}{R}$. By slightly adjustment of the ratio might get a desired high performance shell structure.

It must point out here that although we can find the particular solution by Maple, however, its analytical expression can not be obtained due to the fact that integration of the Heun's function can not be expressed in any special functions, which unfortunately decreases the value of the complex form of toroidal shells. Nevertheless, approximation and or numerical treatment of the particular solution can still be possible because it has been obtained and represented by the Heun's function even in its integration form.

Besides the integration difficulties, the complex form governing equations of toroidal shells and even general shells have some disadvantages, such as they are not able to deal with dynamical and buckling problems. This definitely limits the use of the complex form modelling. To avoid the issues of integration difficulty and others, the displacement type equations must be used as Sun [17], where the closed-form solution for displacement type equation of slender toroidal shells has been obtained. However, the displacement solution for the arbitrary toroidal shells is still an open problem, which therefore is the task for the future.

[1] Reissner, H., Spannungen in Kugelschalen (Kuppeln). Festschrift Heinrich Müller-Breslau (A. Kröner, Leipzig, 1912), pp. 181-193

[2] Meissner, E.,Uber und Elastizitat Festigkeit dunner Schalen, Viertelschr. D. nature.Ges., Bd.60, Zurich (1915).

[3] Wissler, H., Festigkeiberechung von Ringsflachen, Promotionarbeit, Zurich (1916). 
[4] Zhang, W., Toroidal shells, Sci. Rep Nat. Tsinghua Univ., Ser A. 259-349 (1949).

[5] Clark, R.A., On the theory of thin elastic toroidal shells, J. Mech. Phys., Vol.29, No.3, 1950.

[6] Dahl, N.C., Toroidal-shell expansion joints, J. of Applied Mechanics, ASME, 20, 497-503 (1953).

[7] Novozhilov, V.V., The Theory of Thin Shell. (Noordhoff, Groningen, 1959).

[8] Sanders, Jr J.L., An improved first-approximation theory for thin shell, NASA Tech Rep, R-24:1-11, 1959.

[9] Tao, L.N., On toroidal shells, J. of Math and Physics, 38, 130-134 (1959).

[10] Steele, C. R., Toroidal shells with nonsymmetric loading. Ph.D. Dissertation, Stanford University, (1959).

[11] Qian, W.Z. and Liang S.C., Complex form equation and asymptotic solution, J. of Tsinghua University, 19(1), 27-47, 1979.

[12] Qian, W.Z., Papers in Applied Math and Mechanics, Jiangsu Science and Technology Press, 1979.

[13] Xia, Z. H. and Zhang W., The general solution for thin-walled curved tubes with arbitrary loadings and various boundary conditions. Int. J. Pressures and Piping 26, 129-144, 1986.

[14] Zhang, W., Ren W. and Sun B.H., Toroidal Shells - history, current situation and future, Fifth Conf. of Space Structures, Lanzhou, China 1990.

[15] Zhang, R. J. and Zhang, W., Turning point solution for thin toroidal shell vibrations. Int. J. Solids Structures 27(10), 1311-1326, 1991.

[16] Zhang, R.J. and Zhang W., Toroidal shells under Nonsymmetric Loading, Int. J. Solids Structures Vol. 31, No. 19. pp. 2735-2750, 1994.

[17] Sun, B.H., Closed-form solution of axisymmetric slender elastic toroidal shells, Journal of Engineering Mechanics, Vol.136, No.10: 1281-1288 (2010).

[18] Audoly, B. and Pomeau, Y., Elasticity and Geometry - From hair curls to the non-linear response of shells. University of Cambridge, Cambridge, 2010

[19] Sun, B.H., Toroidal Shells, (Nova Novinka, New York, 2012)

[20] Sun B.H., Exact solution of Qian's equation of slender toroidal shells. Mechanics in Engineering, 2016, 38(5): 567-569

[21] Ronveaux, A. ed. Heun's Differential Equations. Oxford University Press, 1995.

[22] Marsden, J.E., Sirovich, L., and Antman, S.S. eds. Hypergeometric Functions and Their Applications. Texts in Applied Mathematics. 56. New York: Springer-Verlag, 1991.

[23] McLachlan, N. W. Theory and Applications of Mathieu Functions. Oxford University Press.

[24] https://www.maplesoft.com/support/help/Maple/view.aspx?path=Mathieu

[25] Marple https://www.maplesoft.com/ 